\title{
Stroke subtypes in Sri Lanka - a hospital based study
}

\author{
S B Gunatilake', B A S Jayasekera² and A P Premawardene ${ }^{3}$ \\ (Index words: CT scanning, cerebral infarction, intracerebral haemorrhage, subarachnoid haemorrhage).
}

\begin{abstract}
Aim Data on stroke subtypes in Sri Lanka are limited, mainly due to the unavailability of brain imaging facilities in most government hospitals. In two leading private hospitals in Colombo, a high proportion of stroke patients have computerised tomography (CT) scanning. Hence we studied stroke patients admitted to these two hospitals to determine the stroke subtypes.
\end{abstract}

Methods A prospective study of 103 consecutive first ever stroke patients who were under the care of the first author during the period 15 May 1995 to 30 August 1996 were studied. Diagnosis of stroke was made according to the WHO definition, and transient ischaemic attacks (TIA) were not included. The pathological subtype was confirmed by CT scan in 99 patients.

Results The age of the patients ranged from 37 to 94 years (mean 64.5). 87 patients were over the age of 50 years. The proportion of pathological subtypes confirmed by CT scanning was cerebral infarction (CI) $74.7 \%$, intracerebral haemorrhage (ICH) $19.1 \%$ and subarachnoid haemorrhage (SAH) $62.2 \%$. Of the infarcts 31 (42\%) were cortical, 30 (41\%) lacunar, $12(16 \%)$ cerebellar and brainstem, and 1 (1.3\%) was a border zone infarct.

Conclusion Direct comparisons with stroke subtypes seen in other countries are not valid due to differences in methodology. In developed countries in the West cerebral infarcts account for about $80 \%$ of all first ever strokes and of these 13 to $21 \%$ are lacunar strokes. Countries in the East (eg. Japan and Hong Kong) have reported a higher proportion of haemorrhages ( $27 \%$ of first ever strokes in Hong Kong). In Sri Lanka the proportion of stroke subtypes seem to be intermediate between these countries in the West and East. Lacunar strokes are commoner in Sri Lanka than in other countries.

\section{Introduction}

Strokes are a major cause of disability and death worldwide and an important health problem in Sri Lanka. There is little information about stroke subtypes from countries in south Asia. Studies from China, Japan and Hong Kong show a higher rate of cerebral haemorrhages when compared with studies from the West $(1,2,3)$. Such data are important for diagnostic, therapeutic and preventive purposes. Data on stroke subtypes in Sri Lanka are not available, the reason for this being the unavailability of brain imaging facilities in most hospitals in the country. In two leading private hospitals in Colombo, a high proportion of stroke patients have computerised tomography (CT) scanning.

\section{Methods}

This prospective study was carried out in two hospitals and includes 103 consecutive first ever strokes admitted during the period from 15 May 1995 to 30 August 1996. Of these 99 had CT scans of the head. Stroke was defined as rapidly developing clinical symptoms or signs of focal or global loss of cerebral function, with symptoms lasting more than 24 hours or leading to death, with no apparent cause other than that of vascular origin (4). Transient ischaemic attacks (TIA) were not included in the study. Strokes were classified into three pathological subtypes (3):

(i) Cerebral infarct. Stroke with CT evidence of infarction in a region compatible with the clinical features or stroke with a normal CT.

(ii) Cerebral haemorrhage. Stroke with CT evidence of parenchymal haemorrhage unrelated to tumour or trauma, in a region compatible with the clinical features.

(iii) Subarachnoid haemorrhage. Strokes with a typical history of acute onset of headache and meningism, not associated with trauma, with a CT scan which shows subarachnoid blood. Cases with the clinical features of subarachnoid haemorrhage (SAH) but without confirmatory investigations (because of early death) were diagnosed as probable subarachnoid haemorrhage.

Cerebral infarcts were further classified into cortical, lacunar, cerebellar and brain stem, and border zone. Clinical features and relevant CT findings were used to achieve this. When the CT did not show a relevant abnormality, as happens when the lesion is too small (as in lacunar and brainstem infarcts), and when the scan has been carried out too early, the clinical features were used to make the subtype classification. Cortical infarcts were diagnosed when the CT showed a cortical infarct or a subcortical lesion larger than $1.5 \mathrm{~cm}$ or when the CT was normal in patients who had higher function deficits such as asphasia, apraxia and visual field defects. Lacunar infarcts were diagnosed in patients who had lacunar syndromes and appropriate CT lesions or normal CT. 


\section{Results}

Our study included 99 patients who had CT scans whose age ranged from 37 to 94 years (mean 64.5, SD 12.4 years). There were 59 men (mean age 65, SD 12.7 years) and 40 women (mean age 63.7, SD 12 years). 34 (34\%) had a history of hypertension, 25 (25\%) of diabetes mellitus, and $4(4 \%)$ had atrial fibrillation. Only one patient gave a history of a recent TIA. $46(46.4 \%)$ of the patients were admitted to hospital within 3 hours of onset of stroke symptoms, 51 (51.5\%) within 6 hours of onset of symptoms. 54 (54.5\%) of the patients were treated initially in the intensive care unit. CT scans were done on the same day in $23 \%$. Of the 99 patients, $74(74.7 \%)$ had cerebral infarcts (CI), $19(19.1 \%)$ had intracerebral haemorrhage (ICH) and 6 (6.2\%) had subarachnoid haemorrhage. Of the cerebral infarcts $31(42 \%)$ were cortical, $30(41 \%)$ were lacunar, 12 were brainstem and cerebellar, and 1 was a border zone infarct. Of the $19 \mathrm{ICH}$ one was a cerebellar haemorrhage.

\section{Discussion}

There is a paucity of stroke data from developing countries. The ideal studies should be community based and have all diagnoses verified by CT or MRI scanning. This is impossible to achieve in developing countries like Sri Lanka. Due to limited CT facilities, stroke patients admitted to government hospitals, including the National Hospital in Colombo, which is a tertiary referral centre, are not subjected to CT scanning routinely. In two major private hospitals in Colombo, over $95 \%$ of the stroke patients admitted are scanned. Patients admitted to private hospitals are from a different socio-economic background, but we believe that data from such study could provide acceptable information regarding stroke subtypes.

Hospital based studies are likely to underestimate mild strokes which are usually cerebral infarcts, rapidly lethal strokes which are usually SAH, and large intracerebral haemorrhages. They also have a tendency to include more haemorrhages. This is because patients with cerebral haemorrhages are more likely to have more moderate to severe strokes than cerebral infarcts, and are more likely to seek admission to hospital. In this study, intracerebral haemorrhage comprised $19.1 \%$. Though direct comparisons with other studies may not be valid due to different methodologies used, the proportion of ICH in this study seems to be higher than those seen in the West $(3,5)$ and lower than those from the East $(2,6)$. In developed countries in the West ICH account for about 15 to $20 \%$ of all strokes, and in the East about 27 to $35 \%$. Being a hospital based study and we assume that proportionately more ICH are included, then the real proportion of ICH could be lower. Of the cerebral infarcts $41 \%$ were lacunar. This is higher than in any other study reported so far. It is possible that poor control of hypertension and intracranial occlusive vascular disease specific to Asians are responsible for this finding (7), but the incidence of hypertension was not high in our patients. If hypertension was a significant factor, it would have resulted in a higher proportion of $\mathrm{ICH}$ as well. An intrinsic intracranial occlusive disease is the more likely explanation for the increased number of lacunar infarcts and this needs further study.

The slight excess of ICH seen in this study may be explained by the fact that this study included only hospitalised patients. What is more intriguing is the clear excess of lacunar strokes among our patients. Management of lacunar strokes differs from that of cardio-embolic, artery to artery embolic and in situ large artery thrombotic strokes. Hence, further studies are necessary to confirm this finding as this would have major implications in the management of stroke patients in Sri Lanka.

\section{References}

1. Tanaka $H$, Ueda $Y$, Date C, Baba L, et al. Incidence of stroke in Shibata, Japan: 1976-1978. Stroke 1981; 12: 460-6.

2. Kay R, Woo J, Kreel L, Wong H Y, Teoh R, Nicholls M G. Stroke subtypes among Chinese living in Hong Kong: The Shatin Stroke Registry. Neurology 1992; 42: 985-7.

3. Bamford J, Sandercock P, Dennis M, Burn J, Warlow C P. A propective study of acute cerebrovascular disease in the community: the Oxfordshire Community Stroke Project 1981-86 (2). Incidence, case fatality rate and overall outcome at one year of cerebral infarction, primary intracerebral haemorrhage and subarachnoid haemorrhage. Journal of Neurology, Neurosurgery and Psychiatry 1990; 53: 16-22.

4. Hatano S. Experience from a multicentre stroke register: a preliminary report. Bulletin WHO 1976; 54: 541-3.

5. Bogousslavsky J, Van melle G, Regli F. The Lausanne stroke registry: analysis of 1000 consecutive patients with first stroke. Stroke 1988; 19: 1083-92.

6. Suzuki K, Kutsuzawa T, Takita K, Ito M, et al. Clinico-epidemiologic study of stroke in Akita, Japan. Stroke 1987; 16: 402-6.

7. Feldman E, Daneault N, Kwan E, Ho KJ, et al. Chinese - white differences in the distribution of occlusive cerebrovascular disease. Neurology 1990; 40: 1541-5. 